\title{
Photometrische Bestimmung des Aluminiums mit Aluminon in Abwässern. Störung durch Chrom und deren Behebung
}

(Beiträge zur chemischen Wasser- und Abwasseranalyse, Nr. 6)

Von H. R. Hegr und E. JueL ${ }^{1}$ )

Eidg. Anstalt für Wasserversorgung, Abwasserreinigung und Gewässerschutz an der Eidg. Technischen Hochschule, Zürich

Direktion: Prof. Dr. O. Jaag

Manuskript eingegangen am 16. Januar 1968

\section{Einleitung}

Das Aluminium wird in Wässern und Abwässern in den meisten Laboratorien photometrisch mit Aluminon bestimmt; entsprechende Arbeitsvorschriften sind in Handbüchern und in der Zeitschriftenliteratur über Abwasseranalytik zu finden [3, 5-7]. Wird die Bestimmung von Aluminium mit Aluminon in gewerblichen und industriellen Abwässern vorgenommen, so ist der Möglichkeit der Störung durch andere Metalle Beachtung zu schenken. Tatsächlich wird die Bestimmung durch einige Schwermetalle, vor allem durch Chrom, erheblich beeinflusst. Chrom stört, indem es in ähnlicher Weise wie Aluminium mit Aluminon einen roten Farblack bildet. Aluminium- und Chromverbindungen können beispielsweise in Abwässern, die bei der anodischen Oxydation zur Oberflächenbehandlung des Aluminiums anfallen (EloxalVerfahren) gleichzeitig vorhanđen und zu bestimmen sein. Nach ECKERT [2] lässt sich eine Reihe störender Metalle, nämlich Eisen, Kupfer, Nickel, Kobalt, Mangan, durch Extraktion mit Natrium-diäthyldithiocarbamat entfernen; er weist aber darauf hin, dass die gleichzeitige Anwesenheit von Chrom eine zusätzliche Trennung erfordern würde. Craft und Makepeace [1] liessen Chrom(III) zu Chrom(VI) oxydieren, wodurch sie bei nicht zu hohen Chromkonzentrationen eine Aufhebung oder weitgehende Verminderung der Störung erreichten; nötigenfalls liess sich das gebildete Chrom(VI) durch Umsetzen zu flüchtigem Chromylchlorid entfernen. Wie wir durch eigene Versuche bestätigt haben, stört Chrom(VI) erheblich weniger als Chrom(III), aber nur dann, wenn keine Thioglykolsäure zugesetzt wird. Luke [4] hat später die Verwendung von Thioglykolsäure vorgeschlagen. Durch diesen Zusatz wird das ebenfalls erheblich störende Eisen(III) zu Eisen(II) reduziert; wir möchten keinesfalls auf die Zugabe von Thioglykolsäure verzichten. Die Thioglykolsäure reduziert aber auch

1) Herrn Georg Wolfensberger sei für die gewissenhafte Ausführung eines grossen Teils der experimentellen Untersuchungen bestens gedankt. 
Chrom(VI) zu Chrom(III). Daher stören Chrom(III) und Chrom(VI), wie wir experimentell festgestellt haben, nach Zugabe von Thioglykolsäure im gleichen Ausmass. Das neben Aluminium vorliegende Chrom muss deshalb vor der Bestimmung entfernt werden. Die Verflüchtigung als Chromylchlorid ist mit erheblicher Entwicklung von Salzsäuredämpfen verbunden. Wir schlagen daher vor, das Chrom(VI), welches in Form der Anionen Chromat und Dichromat gebunden vorliegt, mittels eines Anionenaustauschers zu entfernen. Liegt das Chrom in der dreiwertigen Stufe vor, so muss es vorher zu Chrom(VI) oxydiert werden, was auch notwendig wäre, wenn man es als Chromylchlorid entfernen wollte. Nachstehend geben wir eine Arbeitsvorschrift zur Entfernung des störenden Chroms an; zur anschliessenden Aluminiumbestimmung haben wir die Vorschrift der Standard Methods [7] übernommen.

\section{Arbeitsvorschrift}

\subsection{Reagenzien, besondere Geräte}

Anionenaustauschersäule: Als Austauscher lässt sich beispielsweise Dowex $1 \times 10$, 50/100 mesh (trocken), Feuchtigkeitsgehalt 33-39\%, Ionenform $\mathrm{Cl}^{-}$verwenden. Zur Bereitung der Säule ist soviel Austauscher zu verwenden, dass ein Chromatographierrohr von 15-20 mm innerem Durchmesser 70-150 mm hoch mit Austauscher gefüllt wird (die Höhe der Säule richtet sich nach der voraussichtlichen Häufigkeit der vorzunehmenden Bestimmungen). Der Austauscher ist nötigenfalls vorerst in einem Becherglas über Nacht in entsalztem Wasser quellen zu lassen. Nach dem Einfüllen des Austauscherharzes in das Chromatographierrohr werden etwa $100 \mathrm{ml} n$-Salzsäure durchlaufen gelassen, um den Austauscher von möglicherweise anhaftenden Schwermetallverbindungen zu befreien; hierauf wird mit entsalztem Wasser nachgespült. Eine einzelne Säule kann für eine grössere Anzahl von Bestimmungen verwendet werden; wenn der Austauscher erschöpft ist, empfiehlt es sich, die Harzfüllung zu verwerfen.

Aluminiumstammlösung: 0,500 g Aluminiummetall (z. B. Aluminiumband oder -späne, mit mindestens 99,9\% Al) werden in $10 \mathrm{ml}$ konz. Salzsäure p.a. unter leichtem Erwärmen gelöst. Um vollständiges Auflösen zu erreichen, müssen vielleicht nochmals einige Milliliter Salzsäure zugegeben werden. Mit entsalztem Wasser wird in einem Messkolben auf $1000 \mathrm{ml}$ verdünnt. $1 \mathrm{ml}$ Stammlösung enthält 0,5 mg Al.

Aluminiumgebrauchslösung: $5,00 \mathrm{ml}$ der Aluminiumstammłösung werden mit entsalztem Wasser auf $250 \mathrm{ml}$ verdünnt. $1 \mathrm{ml}$ Gebrauchslösung enthält $0,01 \mathrm{mg} \mathrm{Al}$. Die Gebrauchslösung ist bei Bedarf täglich frisch zu bereiten.

p-Nitrophenollösung: $1 \mathrm{~g}$ p-Nitrophenol wird in entsalztem Wasser zu $100 \mathrm{ml}$ gelöst. $n$-Salzsäure.

$0,01 n$-Salzsäure.

Ammoniumhydroxidlösung: $200 \mathrm{ml}$ konz. Ammoniak p.a. $(d=0,910)$ werden mit entsalztem Wasser zu $1000 \mathrm{ml}$ verdünnt.

Thioglykolsäurelösung: $1 \mathrm{ml}$ Thioglykolsäure, 80-98prozentig, wird mit entsalztem Wasser zu $100 \mathrm{ml}$ verdünnt. Die Lösung ist nur wenige Tage lang haltbar.

Aluminonpufferlösung: Folgende Reagenzien werden für sich in je etwa $100 \mathrm{ml}$ entsalztem Wasser gelöst und die erhaltenen Lösungen gemischt: 133 g Ammonium- 
azetat, $126 \mathrm{ml}$ konz. Salzsäure p. a., 0,9g Aluminon (Aurintrikarbonsäure-Ammoniumsalz) und $10 \mathrm{~g}$ arabisches Gummi. Die Mischung wird mit entsalztem Wasser auf $1000 \mathrm{ml}$ verdünnt, über Nacht stehengelassen und durch Glaswolle oder besser durch ein grosser Glasfilter (z.B. Schott G 2), ohne zu saugen, filtriert. Das Filtrat kann noch etwas trübe sein; die Trübung bleibt beim weiteren Stehenlassen in Suspension. Das pH der Lösung soll zwischen 3,8 und 4,0 liegen; sie ist etwa 6 Monate lang haltbar.

Kaliumpermanganatlösung: $4 \mathrm{~g}$ Kaliumpermanganat p.a. werden in $100 \mathrm{ml}$ entsalztem Wasser gelöst.

Natriumazidlösung: 0,5 $\mathrm{g}$ Natriumazid $\left(\mathrm{NaN}_{3}\right)$ werden in $100 \mathrm{ml}$ entsalztem Wasser gelöst.

Schwefelsäure 1+1: 1 Volumenteil Schwefelsäure konz. p.a. zu 1 Volumenteil entsalztem Wasser zugeben (Vorsicht, Erwärmung!).

\subsection{Probenkonservierung}

Die Proben sind, falls sie nicht deutlich sauer reagieren, durch Zugabe eines gemessenen Volumens $n$-Salzsäure schwach anzusäuern, damit ein Ausflocken von Aluminium bis zur Untersuchung mit Sicherheit vermieden wird. In der Regel genügen $0,5 \mathrm{ml} n$-Salzsäure auf $25 \mathrm{ml}$ Probe.

\subsection{Oxydation von Chrom $(I I I)$ zu Chrom(VI)}

Liegt das Chrom ganz oder teilweise als Chrom(III) vor, so ist es vor seiner Entfernung (nach 2.4.) zu Chrom(VI) zu oxydieren. Falls das Chrom(III) ebenfalls bestimmt werden muss, empfiehlt es sich, dieses als Differenz zwischen dem gesamten Chrom und dem Chrom(VI) zu ermitteln. Zu diesem Zweck wird das Chrom(VI) für sich und in einem weiteren Ansatz das gesamte Chrom nach Oxydation des Chrom(III) zu Chrom(VI) photometrisch mit Diphenylcarbazid bestimmt. Die Oxydation kann dann für die Chrom(III)-Bestimmung und als vorbereitende Operation für die Entfernung des Chrom(III) bei der Aluminiumbestimmung gemeinsam durchgeführt werden: $200 \mathrm{ml}$ Probe werden mit $4 \mathrm{ml}$ Schwefelsäure $1+1$ versetzt und zum Sieden erhitzt. Es werden 10 Tropfen Kaliumpermanganatlösung zugegeben. Falls die Violettfärbung während des weiteren Siedens ausbleicht, ist die Zugabe von Kaliumpermanganatlösung zu wiederholen; es muss eine kräftige Violettfärbung noch nach 2 Minuten langem Sieden bestehen bleiben. Hierauf werden $5 \mathrm{ml}$ Natriumazidlösung zugegeben und die Mischung wiederum zum leichten Sieden erhitzt. Falls die Rotfärbung nach 30 Sekunden langem Sieden nicht völlig verschwindet, ist nochmals möglichst wenig Natriumazidlösung zuzugeben. Das Sieden ist bis 1 Minute nach dem vollständigen Verschwinden der Färbung fortzusetzen. Ein möglicherweise gebildeter Niederschlag von Mangandioxid ist durch Glaswolle oder durch ein Glasfilter abzufiltrieren. Das Filter ist mit möglichst wenig entsalztem Wasser nachzuwaschen. Die wenn nötig filtrierte Lösung wird mit entsalztem Wasser auf $200 \mathrm{ml}$ ergänzt.

Zur Ermittlung des Gesamtchrom-bzw. des Chrom(III)-Gehaltes kann von der nach der Oxydation erhaltenen Lösung ein aliquoter Teil verwendet werden.

\subsection{Entfernung des Chroms}

Unmittelbar vor dem Perkolieren jedes einzelnen Probenansatzes werden $50 \mathrm{ml}$ 0,01n-Salzsäure durch die Anionenaustauschersäule fliessen gelassen. 
Bei Proben, von denen man weiss, dass das Chrom gesamthaft als Chrom(VI) vorliegt, kann die mit Salzsäure konservierte Probe direkt (ohne Oxydation nach 2.3) auf die Anionenaustauschersäule gegeben werden. Wurde die Oxydation nach 2.3 vorgenommen, so ist die nach 2.3 erhaltene Lösung auf die Anionenaustauschersäule zu geben.

Man lässt 50-100 ml durch die Anionenaustauschersäule fliessen, wobei die ersten $25 \mathrm{ml}$ des Perkolates verworfen werden. Die durch Chrom(VI) gegebenenfalls erzeugte Gelbfärbung darf im Perkolat nicht mehr feststellbar sein.

\subsection{Aluminiumbestimmung}

Vor Perkolat wird ein Volumen, das $0-0,03 \mathrm{mg} \mathrm{Al}$ enthält $(25 \mathrm{ml}$, gegebenenfalls ist ein kleineres Volumen mit entsalztem Wasser auf $25 \mathrm{ml} \mathrm{zu}$ ergänzen), in ein weites dickwandiges Reagenzglas eingemessen. Gleichzeitig werden eine Blindprobe und Standardlösungen bis zu $0,03 \mathrm{mg} \mathrm{Al}$ angesetzt. Zum Perkolat wird 1 Tropfen $p$-Nitrophenollösung zugesetzt und dann Ammoniumhydroxidlösung, bis eben eine Gelbfärbung auftritt. Dann wird tropfenweise $n$-Salzsäure zugegeben, bis die Gelbfärbung des $p$-Nitrophenols gerade verschwindet. Zu Probenansatz, Blindprobe und Standards werden je $1 \mathrm{ml}$ Thioglykolsäurelösung zugegeben und gemischt. Unter Umrühren werden ferner 5,0 ml Aluminonpufferlösung zugesetzt. Dann werden alle Ansätze in ein siedendes Wasserbad gestellt und vom Moment an, da die Temperatur in einem weiteren, mit $30 \mathrm{ml}$ Wasser versetzten Reagenzglas $90^{\circ} \mathrm{C}$ erreicht hat, während 15 Minuten erhitzt. Der Spiegel des Wassers im Wasserbad muss oberhalb des Flüssigkeitsspiegels der Ansätze in den Reagenzgläsern stehen. Die Ansätze werden hierauf in Wasser auf etwa $20^{\circ} \mathrm{C}$ abgekühlt, in 50-ml-Nesslerzylinder übergeführt und mit entsalztem Wasser bis zur Marke verdünnt. Die Farbmessung erfolgt bei $525 \mathrm{~nm}$ oder mit einem entsprechenden Filter. Das Lambert-Beersche Gesetz wird im vorgesehenen Konzentrationsbereich nur annähernd erfüllt.

\section{Bemerkungen}

Anstelle der Aluminiumbestimmung nach der Vorschrift unter 2.5 kann selbstverständlich eine andere Arbeitsvorschrift angewandt werden.

Die Geschwindigkeit, mit welcher der Probenansatz durch die Austauschersäule fliesst, wird beim Arbeiten nach der Vorschrift vor allem durch die Säulenhöhe bestimmt und gewährleistet in der dadurch gegebenen Begrenzung die Vollständigkeit der Abtrennung. Bei einer Säulenhöhe von $150 \mathrm{~mm}$ betrug die Durchflussgeschwindigkeit etwa $8 \mathrm{ml}$ in der Minute.

\section{Experimentelle Untersuchungen zur Arbeitsvorschrift}

Bei der Ausführung hielten wir uns, sofern nichts anderes vermerkt ist, an die unter 2. angegebene Arbeitsvorschrift. Die Messungen erfolgten mit dem Photometer Eppendorf, Filter 546, 10-mm-Küvette. Chrom(III) wurde als Kaliumchrom(III)sulfat zugegeben, Chrom(VI) als Kaliumchromat. Beim Ansetzen der Testlösungen wurde von der Voraussetzung ausgegangen, dass das Probeneinmass $25 \mathrm{ml}$ beträgt, 
d.h., es wurde ein Vierzigstel der Literkonzentrationen an Aluminium und Chrom eingesetzt.

4.1 Keine Vorbehandlung nach 2.3 und 2.4, Aluminiumstandards

\begin{tabular}{lll}
\hline mg A1/1 & Extinktion & \\
\hline 0,5 & 0,162 & 0,152 \\
1,0 & 0,319 & 0,298 \\
2,0 & 0,604 & 0,584 \\
\hline
\end{tabular}

4.2 Keine Vorbehandlung nach 2.3 und 2.4, Aluminium-Chrom-Testlösungen

\begin{tabular}{llllll}
\hline $\operatorname{mg~A1} / 1$ & $\mathrm{mg} \operatorname{Cr}(\mathrm{III}) / 1$ & Extinktion & $\mathrm{mg} \mathrm{Al} / 1$ & $\operatorname{mg~Cr}(\mathrm{III}) / 1$ & Extinktion \\
\hline 0 & 0 & - & 2,0 & 0 & 0,596 \\
0 & 2,0 & 0,315 & 2,0 & 2,0 & 0,750 \\
0 & 4,0 & 0,450 & 2,0 & 4,0 & 0,872 \\
0 & 10,0 & 0,732 & 2,0 & 10,0 & 1,020 \\
0 & 20,0 & 0,975 & 2,0 & 20,0 & 1,140 \\
\hline
\end{tabular}

Der Versuch zeigt die erhebliche positive Störung durch Chrom(III).

4.3 Keine Vorbehandlung nach 2.3 und 2.4, Chromtestlösungen ohne Aluminium, mit und ohne Thioglykolsäure

\begin{tabular}{lllll}
\hline $\mathrm{mg} \mathrm{Al} / \mathrm{l}$ & $\mathrm{mg} \mathrm{Cr}(\mathrm{III}) / \mathrm{I}$ & $\mathrm{mg} \operatorname{Cr}(\mathrm{VI}) / \mathrm{l}$ & Extinktion $\mathrm{I}$ & Extinktion II \\
\hline 0 & 0,5 & 0 & 0,096 & 0,091 \\
0 & 1,0 & 0 & 0,183 & 0,164 \\
0 & 2,0 & 0 & 0,345 & 0,297 \\
0 & 0 & 0,5 & 0,110 & 0,059 \\
0 & 0 & 1,0 & 0,148 & 0,067 \\
0 & 0 & 2,0 & 0,283 & 0,083 \\
\hline
\end{tabular}

Extinktion I: bei Thioglykolsäurezugabe nach Vorschrift.

Extinktion II: bei Unterlassen der Thioglykolsäurezugabe.

Extinktion II durch Messung gegen eine Blindprobe ohne Thioglykolsäurezugabe ermittelt.

Der Versuch bestätigt, dass die Störung durch Chrom(VI) geringer ist als durch Chrom(III), dass aber bei der notwendigen Thioglykolsäurezugabe infolge der Reduktion des Chrom(VI) zu Chrom(III) das Ausmass der Störung in beiden Wertigkeitsstufen gleich ist. 
4.4 Vorbehandlung nach 2.3 und 2.4, Aluminiumstandards, ferner Aluminium-Chrom-Testlösungen

\begin{tabular}{lcc}
\hline $\operatorname{mg~A1} / 1$ & $\operatorname{mg} \mathrm{Cr}(\mathrm{III}) / 1$ & Extinktion \\
\hline 0,5 & 0 & 0,170 \\
1,0 & 0 & 0,334 \\
2,0 & 0 & 0,642 \\
1,0 & 1,0 & 0,341 \\
1,0 & 5,0 & 0,335 \\
1,0 & 20,0 & 0,339 \\
2,0 & 1,0 & 0,628 \\
2,0 & 5,0 & 0,681 \\
2,0 & 20,0 & 0,642 \\
\hline
\end{tabular}

Die Ergebnisse zeigen, dass bei der vorgeschriebenen Vorbehandlung keine Verluste an Aluminium auftreten und anderseits das Chrom vollständig abgetrennt wird. Sie zeigen auch, dass das vorgeschriebene Perkolieren von $50 \mathrm{ml} 0,01 n$-Salzsäure durch die Austauschersäule und das Verwerfen der ersten $25 \mathrm{ml}$ des Perkolates genügen, um das Verschleppen von Aluminium von einem Probenansatz zum nächsten zu verhindern.

\subsection{Beobachtungen}

Bei der Oxydation von Chrom(III) zu Chrom(VI) (vgl. Arbeitsvorschrift 2.3) bildet sich in der Regel Mangandioxid, das aber beim Sieden nach der Zugabe von Natriumazid meistens in Lösung geht.

Auch beim oft wiederholten Durchlaufenlassen von Chrom(VI)-haltigen Lösungen durch die Anionenaustauschersäule blieb das Chrom(VI) ausschliesslich im oberen Teil der Säule haften, was an dessen Gelbfärbung erkenntlich war. Wurde die Anionenaustauschersäule nach Gebrauch längere Zeit unbenützt stehengelassen, so färbte sie sich unterhalb der gelben Zone grünblau, sicher infolge der teilweisen Reduktion von Chrom(VI) zu Chrom(III) auf der Säule. Im Gegensatz zum gelben Chrom(VI) lässt sich das Chrom(III) mit verdünnter, beispielsweise $0,01 n$-Salzsäure sehr leicht auswaschen, weshalb unter 2.4 vorgeschrieben ist, vor dem Perkolieren der Proben $0,01 n$-Salzsäure durch die Säule fliessen zu lassen.

\section{Zusammenfassung}

Es wird eine Arbeitsvorschrift zur Bestimmung des Aluminiums in Abwässern, die gleichzeitig Chrom enthalten, angegeben. Das störende Chrom wird zu Chrom(VI) oxydiert, sofern es nicht bereits in dieser Form vorliegt, und mittels eines Anionenaustauschers aus der Lösung entfernt. Hierauf wird das Aluminium photometrisch mit Aluminon bestimmt. 


\section{SUMMARY}

A method is described for the determination of aluminum in waste waters which also contain chromium. The interfering chromium, if not already present in the sixvalent state, is oxidized to chromium(VI) and is removed from the solution by means of an anion exchanger. Afterwards the aluminum is determined photometrically with aluminon.

\section{LITERATURVERZEICHNIS}

[1] Craft C. H., Makepeace G. R., Colorimetric Estimation of Aluminum in Aluminum Steel, Ind. Engng Chem. analyt. Edn 77, 206 (1945).

[2] Eckert G., Analyse von Nickellegierungen für Oxydkathoden, 3. Mitteilung: Die Bestimmung des Aluminiums, Z. analyt. Chem. 153, 261 (1956).

[3] GIEBLer G., Vergleichende Untersuchungen der zur Bestimmung von Aluminiumionen im Wasser gebräuchlichen Methoden, Z. analyt. Chem. 184, 401 (1961).

[4] Luke C. L., Photometric Determination of Aluminum in Lead, Antimony, and Tin and Their Alloys, Analyt. Chem. 24, 1122 (1952).

[5] Rodier J., L'analyse chimique et physico-chimique de l'eau, 3. Aufl., 216 (Dunod, Paris 1966).

[6] SHULl K. E., Suggested Modified Aluminon Method for Aluminum Determination, J. Am. Wat. Wks Ass. 52, 779 (1960).

[7] Standard Methods for the Examination of Water and Wastewater, 12. Aufl., 53 (Am. Public Health Assoc., New York 1965).

Adresse der Autoren:

Dr. H. R. Hegi und E. Juel, dipl. Ing. chem. ETH, EAWAG,

Physikstrasse 5, 8044 Zürich.

Separatdruck aus der Schweiz. Zeitschrift für Hydrologie 30, Fasc. 1 (1968) Herausgegeben mit Unterstützung der Stiftung der Wirtschaft zur Förderung des Gewässerschutzes in der Schweiz 